Research Article

\title{
Chromatographic Fingerprinting Based on Column Switching Technology for Quality Evaluation of Tianmeng Oral Liquid
}

\author{
Meng Yuan, ${ }^{1}$ Xiaoyan Liu, ${ }^{1}$ Yue Sun, ${ }^{1}$ Linlin Wang, ${ }^{1}$ Pian Jin, ${ }^{1}$ Xiaoqi Zhuang, \\ Hengchang Zang, ${ }^{1,2,3}$ Zhonghu Zhang $\mathbb{D}^{4},{ }^{4}$ and Lei Nie $\mathbb{B}^{1,3}$ \\ ${ }^{1}$ School of Pharmaceutical Sciences, Cheeloo College of Medicine, Shandong University, Jinan 250012, China \\ ${ }^{2}$ National Glycoengineering Research Center, Jinan 250012, China \\ ${ }^{3}$ National Medical Products Administration Key Laboratory for Technology Research and Evaluation of Drug Products, \\ Shandong University, Jinan 250012, China \\ ${ }^{4}$ Shandong Institute for Food and Drug Control, Jinan 250100, China \\ Correspondence should be addressed to Zhonghu Zhang; 997513594@qq.com and Lei Nie; nielei2004@sdu.edu.cn
}

Received 19 July 2021; Revised 17 August 2021; Accepted 13 September 2021; Published 29 September 2021

Academic Editor: Neil D. Danielson

Copyright ( 2021 Meng Yuan et al. This is an open access article distributed under the Creative Commons Attribution License, which permits unrestricted use, distribution, and reproduction in any medium, provided the original work is properly cited.

\begin{abstract}
Separation power was limited when the conventional high-performance liquid chromatography (HPLC) fingerprinting method based on a single column was used to analyze very complex traditional Chinese medicine (TCM) preparations. In this research, a novel HPLC fingerprinting method based on column switching technology by using a single pump was established for evaluating the quality of Tianmeng oral liquid (TMOL). Twelve batches of TMOL samples were used for constructing HPLC fingerprints. Compared with the 16 common peaks in fingerprinting with a single column, 25 common peaks were achieved with two columns connected through a six-way valve. The similarity analysis combined with bootstrap method was applied to determine the similarity threshold, which was 0.992 to distinguish expired samples and unexpired samples. Principal component analysis (PCA) and hierarchical clustering analysis (HCA) were also applied to classify the TMOL samples, and results revealed that expired and unexpired samples are classified into two categories. The HPLC fingerprinting based on column switching technology with better separation power and higher peak capacity could characterize chemical composition information more comprehensively, providing an effective and alternative method to control and evaluate the quality of TMOL, which would offer a valuable reference for other TCM preparations.
\end{abstract}

\section{Introduction}

Tianmeng oral liquid (TMOL) is a traditional Chinese medicine (TCM) formula preparation composed of seventeen Chinese herbs following the principles of "Jun (emperor)-Chen (minister)-Zuo (adjuvant)-Shi (courier)," which has the effect of replenishing Qi and tonifying kidney, nourishing heart, and calming mind, and commonly used in the treatment of insomnia caused by deficiency of spleen and kidney $[1,2]$. Jun (emperor) includes Acanthopanax senticosus and Polygonatum sibiricum. Chen (minister) includes Rehmannia glutinosa, Lycium chinense, Fructus Mori, Epimedium brevicornu, Silkworm moth, Astragalus membranaceus, and Codonopsis pilosula. Zuo (adjuvant) includes
Wolfiporia cocos, Pericarpium Citri Reticulatae, Rhizoma Pinelliae, and Crataegus pinnatifida. Shi (courier) is Strychnos nux-vomica. For the complex system of TCM, the establishment of its fingerprint is an effective method to evaluate the interbatch consistency and stability of quality and has been internationally recognized [3-5]. TMOL is a compound preparation of TCM with the characteristics of multicomponents and multitargets, and its fingerprint has been established on a single column by high-performance liquid chromatography (HPLC) [6]. In addition, other relevant analytical methods available in the literature for quality evaluation of Tianmeng capsules are primarily based on quantification of a single bioactive component, which is difficult to reflect the quality comprehensively [7-9]. The 
more chromatographic peaks obtained in HPLC fingerprinting could reflect the completer information about chemical components, which is more conducive to reflect the inner quality of TCM comprehensively [10-12]. However, most researches on fingerprinting are based on a single column and the corresponding fingerprint has limited selectivity and separation power to analyze complex TCM preparations [13-15].

The column switching technique is used to connect chromatographic columns with different stationary phases, and the selectivity and resolution of chromatography could be improved to obtain more chromatographic peaks characterizing more chemical information, which is already widely applied to analyze complex systems [16-18]. Usually, the mode of column switching can be divided into twocolumn double-pump system, three-column three-pump system, and complex coupling system [19], while more pumps require more hardware costs. To carry out automatic column switching, the instrument should be equipped with a double-pump infusion system and a high-pressure flow path selection valve at least $[20,21]$. There are few studies on HPLC fingerprinting methods based on column switching of a single pump to obtain more chromatographic peaks for TCM. Given the existing shortcomings, we proposed to establish a single-pump double-column HPLC column switching system by connecting different polarity columns with a six-way valve, which fully combined the advantages of separation selectivity of different polarity chromatographic columns to effectively increase peak capacity, improve the separation effect of characteristic components, and consequently reflect more comprehensive information in fingerprint.

In this study, the column switching technology based on a single pump was employed for the development of the HPLC fingerprinting method to carry out the quality evaluation of TMOL. The present method was compared with a single-column method in different aspects. Various chemometric methods such as similarity analysis, principal component analysis (PCA), and hierarchical clustering analysis (HCA) were all applied to distinguish different batches of TMOL samples for quality evaluation.

\section{Materials and Methods}

2.1. Materials and Reagents. Syringin (purity: $>98 \%$ ) and calycosin 7-O-glucoside (purity: >98\%) were purchased from Ruifensi Biotechnology Co., Ltd. (Chengdu, China). Icariin (purity: $>98 \%$ ), hesperidin (purity: $>98 \%$ ), and chlorogenic acid (purity: >98\%) were purchased from Mansite Biotechnology Co., Ltd. (Chengdu, China). HPLC grade acetonitrile and methanol were obtained from Tedia (Fairfield, USA) and all other solvents used, including acetic acid, phosphoric acid, and formic acid were analytical grade, were purchased from Sinopharm Chemical Reagent (Beijing, China). Deionized water was purified with a Milli-Q water purification system (Molsheim, France). TMOL samples were produced by Rongchang Pharmaceutical Co., Ltd. (Yantai, China) and were purchased from retail pharmacies. The batch numbers
(S1-S12) were 170202, 170204, 170706, 171112, 171121013, $171121021,171218,180101,180103,180104,180117$, and 180203, respectively.

2.2. Column Switching Technology. The HPLC column switching system with a single-pump double-column system was successfully established by adding a six-way injection valve. The two chromatographic columns were connected through a six-way valve. The first kind of connection method is shown in Figures 1(a) and 1(b). The position of the six-way valve in the first $35 \mathrm{~min}$ is $1 \longrightarrow 2$ and the two-column analysis was performed. After $35 \mathrm{~min}$, the six-way valve was switched to $1 \longrightarrow 6$ and the single-column analysis was performed. The second kind of connection method is shown in Figures $1(\mathrm{c})$ and $1(\mathrm{~d})$. The position of the six-way valve in the first $35 \mathrm{~min}$ is $1 \longrightarrow 2$ for single-column analysis. The sixway valve was switched to $1 \longrightarrow 6$ after $35 \mathrm{~min}$, and twocolumn analysis was performed. Samples were injected twice for one sample analysis according to two connection methods, and we focused on the comparison of the singlecolumn method and the two-column method based on column switching technology.

\subsection{Apparatus and Chromatographic Conditions.} Chromatographic separations were carried out by an Agilent 1260 HPLC instrument (Agilent Technologies, USA), including a quaternary pump, an autosampler, a column temperature controller, and a diode array detector, connected to Agilent ChemStation software. The two chromatographic columns employed were an ACE EXCEL $\mathrm{C}_{18}$ column $(250 \times 4.6 \mathrm{~mm}, 5 \mu \mathrm{m})$ and a JADE-PAK $\mathrm{C}_{8}$ column $(50 \times 4.6 \mathrm{~mm}, 3.5 \mu \mathrm{m})$. The mobile phase was comprised of $0.1 \%$ aqueous phosphoric acid $(A)$ and acetonitrile (B). The elution condition was set as follows: $5 \%-5 \%$ B for $0-5 \mathrm{~min}, 5 \%-14 \%$ B for $5-15 \mathrm{~min}, 14 \%-18 \% \mathrm{~B}$ for $15-21 \mathrm{~min}, 18 \%-21 \%$ B for $21-30 \mathrm{~min}, 21 \%-24 \% \mathrm{~B}$ for $30-35 \mathrm{~min}, 24 \%-30 \% \mathrm{~B}$ for $35-40 \mathrm{~min}, 30 \%-36 \% \mathrm{~B}$ for $40-50 \mathrm{~min}, 36 \%-95 \% \mathrm{~B}$ for $50-60 \mathrm{~min}, 95 \%-95 \% \mathrm{~B}$ for 60-65 min, and $95 \%-5 \% \mathrm{~B}$ for $65-75 \mathrm{~min}$. A low rate of $0.6 \mathrm{~mL} / \mathrm{min}$ and a temperature of $25^{\circ} \mathrm{C}$ were maintained. A wavelength used to detect signal was selected as $230 \mathrm{~nm}$ and the injection volume was set as $10 \mu \mathrm{L}$.

\subsection{Preparation of Reference Standard Solution and Sample} Solution. Five kinds of reference substances were dissolved in methanol to prepare mixed reference standard solution, and the final concentration was as follows: calycosin 7-Oglucoside $(0.00150 \mathrm{~g} / \mathrm{mL})$, syringin $(0.00135 \mathrm{~g} / \mathrm{mL})$, icariin $(0.00178 \mathrm{~g} / \mathrm{mL})$, chlorogenic acid $(0.00294 \mathrm{~g} / \mathrm{mL})$, and hesperidin $(0.00225 \mathrm{~g} / \mathrm{mL})$. One TMOL sample $(10 \mathrm{~mL})$ was taken from each batch for analysis. Methanol was added to the samples for ultrasonic extraction for 5 minutes; then the volume of the extract was adjusted to $25 \mathrm{~mL}$. Furthermore, to remove the insoluble components, the centrifugation was performed for $10 \mathrm{~min}$ under the condition of $9668.16 \times \mathrm{g}$ to obtain supernatant, which was filtered with $0.22 \mu \mathrm{m}$ membrane prior to injecting. 


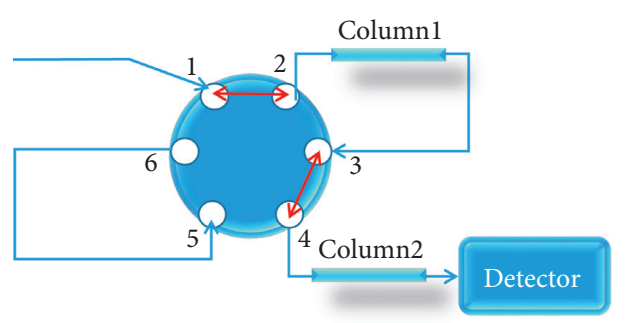

(a)

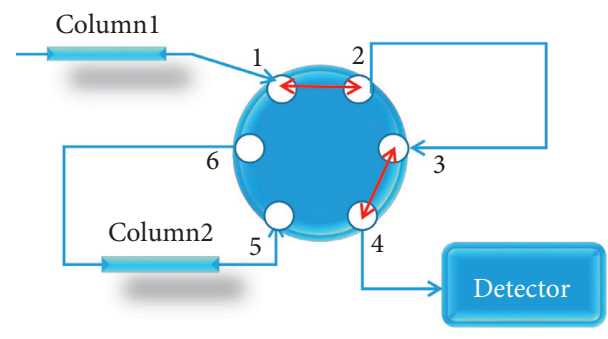

(c)

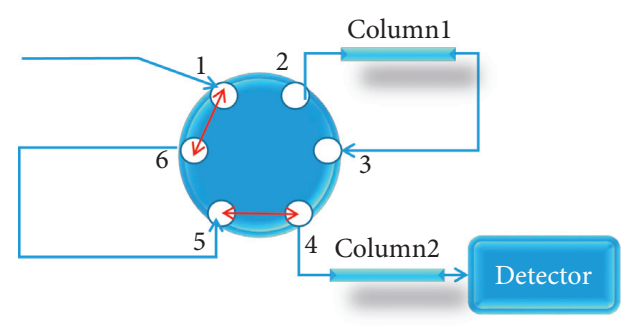

(b)

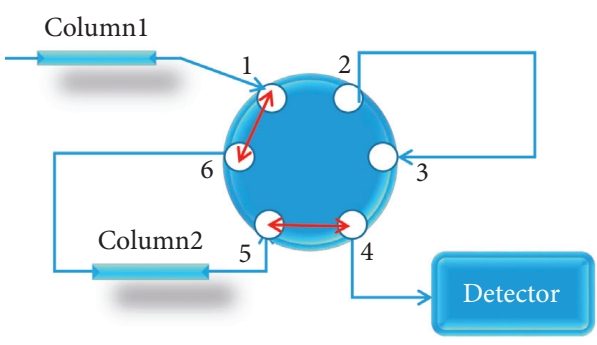

(d)

FIGURE 1: Chromatographic column-connection method of column switching technology based on a single pump.

2.5. Data Analysis. "Similarity Evaluation System for Chromatographic Fingerprint of TCM" (Chinese Pharmacopoeia Commission, 2012A) was applied to analyze fingerprints. The similarity was calculated by the cosine of the angle between the sample fingerprinting and the reference fingerprinting with MATLAB (MathWorks, R2019a). The variability of HPLC fingerprint analysis of 12 batches of samples was evaluated by principal component analysis (PCA) with MATLAB (MathWorks, R2019a). Hierarchical clustering analysis was performed using Origin software (OriginLab, 2021b).

\section{Results and Discussion}

3.1. Optimization of Coupled Columns for Fingerprinting. Choices of chromatographic columns are based on key parameters such as stationary phase and lengths, which have a significant influence on selectivity and resolution. In this research, considering the relatively strong polarity of components of TMOL, the column with a shorter chain length has relatively greater polarity, and so reversed-phase chromatographic columns with different stationary phases and different chain lengths are combined for analysis. The optimal result of two connected columns was determined by trial and error according to the columns available in our research laboratory.

With the purpose of obtaining more chromatographic peaks in the HPLC fingerprinting of TMOL, two columns with different stationary phases, such as Phenomenex Synergi Hydro-RP $\mathrm{C}_{18}(4 \mu \mathrm{m}, 250 \times 4.6 \mathrm{~mm})$ and JADE-PAK $\mathrm{C}_{8}(3.5 \mu \mathrm{m}, 50 \times 4.6 \mathrm{~mm}), \quad \mathrm{ACE}$ EXCEL $\mathrm{C}_{18}(5 \mu \mathrm{m}$, $250 \times 4.6 \mathrm{~mm})$ and JADE-PAK $\mathrm{C}_{8}(3.5 \mu \mathrm{m}, 50 \times 4.6 \mathrm{~mm})$, JADE-PAK $\mathrm{C}_{8}(5 \mu \mathrm{m}, 150 \times 4.6 \mathrm{~mm})$ and JADE-PAK ODSAQ $(5 \mu \mathrm{m}, 150 \times 4.6 \mathrm{~mm})$, and JADE-PAK $\mathrm{C}_{8}(5 \mu \mathrm{m}$, $150 \times 4.6 \mathrm{~mm})$ and Sharpsil- $\mathrm{U} \mathrm{C}_{4}(5 \mu \mathrm{m}, 150 \times 4.6 \mathrm{~mm})$, were connected through a six-way valve. In addition, the combination of ACE EXCEL $\mathrm{C}_{18}(5 \mu \mathrm{m}, 250 \times 4.6 \mathrm{~mm})$ and JADE-PAK C $8(5 \mu \mathrm{m}, 150 \times 4.6 \mathrm{~mm})$ was also tried; that is, the total column length is $400 \mathrm{~mm}$, but the results showed the column pressure increased and the separation effect was not improved significantly. Therefore, we did not try further and the total length of the two columns was limited to $300 \mathrm{~mm}$ to prevent excessive column pressure. As shown in Figure 2, it is found that ACE EXCEL $\mathrm{C}_{18}(250 \times 4.6 \mathrm{~mm}$, $5 \mu \mathrm{m})$ and JADE-PAK $\mathrm{C}_{8}(50 \times 4.6 \mathrm{~mm}, 3.5 \mu \mathrm{m})$ (combination $\mathrm{b}$ ) could get the better peak capacity and resolution. A total of 56 peaks with peak heights greater than $10 \mathrm{mAU}$ were separated by the two columns based on ACE EXCEL $\mathrm{C}_{18}(5 \mu \mathrm{m}, 250 \times 4.6 \mathrm{~mm})$ and JADE-PAK $\mathrm{C}_{8}(3.5 \mu \mathrm{m}$, $50 \times 4.6 \mathrm{~mm}$ ), which was the best among the different column-connection modes, and the resolution of 34 peaks was more than 1.5. Through the first kind of connection method (shown in Figures 1(a) and 1(b)), more polar components can be separated by double-column analysis before $35 \mathrm{~min}$. Through the second kind of connection method (shown in Figures 1(c) and 1(d)), more components with less polarity can be obtained by double-column analysis after $35 \mathrm{~min}$.

\subsection{Optimization of HPLC Chromatographic Conditions.}

The HPLC conditions including mobile phase with different acids, gradient elution, column temperature, flow rate, and detection wavelength were optimized for obtaining fingerprints with satisfactory separation. Acetonitrile was selected as a solvent eluent to obtain good chromatographic behavior and low background noise. Since the addition of acid additives in the mobile phase can reduce the tailing degree of the chromatographic peak and improve the peak shape, the effect of adding different acids $(0.1 \%$ formic acid, $0.1 \%$ acetic acid, and $0.1 \%$ phosphoric acid) into mobile phase $\mathrm{A}$ was investigated, and the results showed that the effect of adding phosphoric acid was the best (Figure S1). By adjusting the proportion of the mobile phase, the baseline was stable, the retention time of each chromatographic peak was moderate, more peaks were obtained, and better separation was 


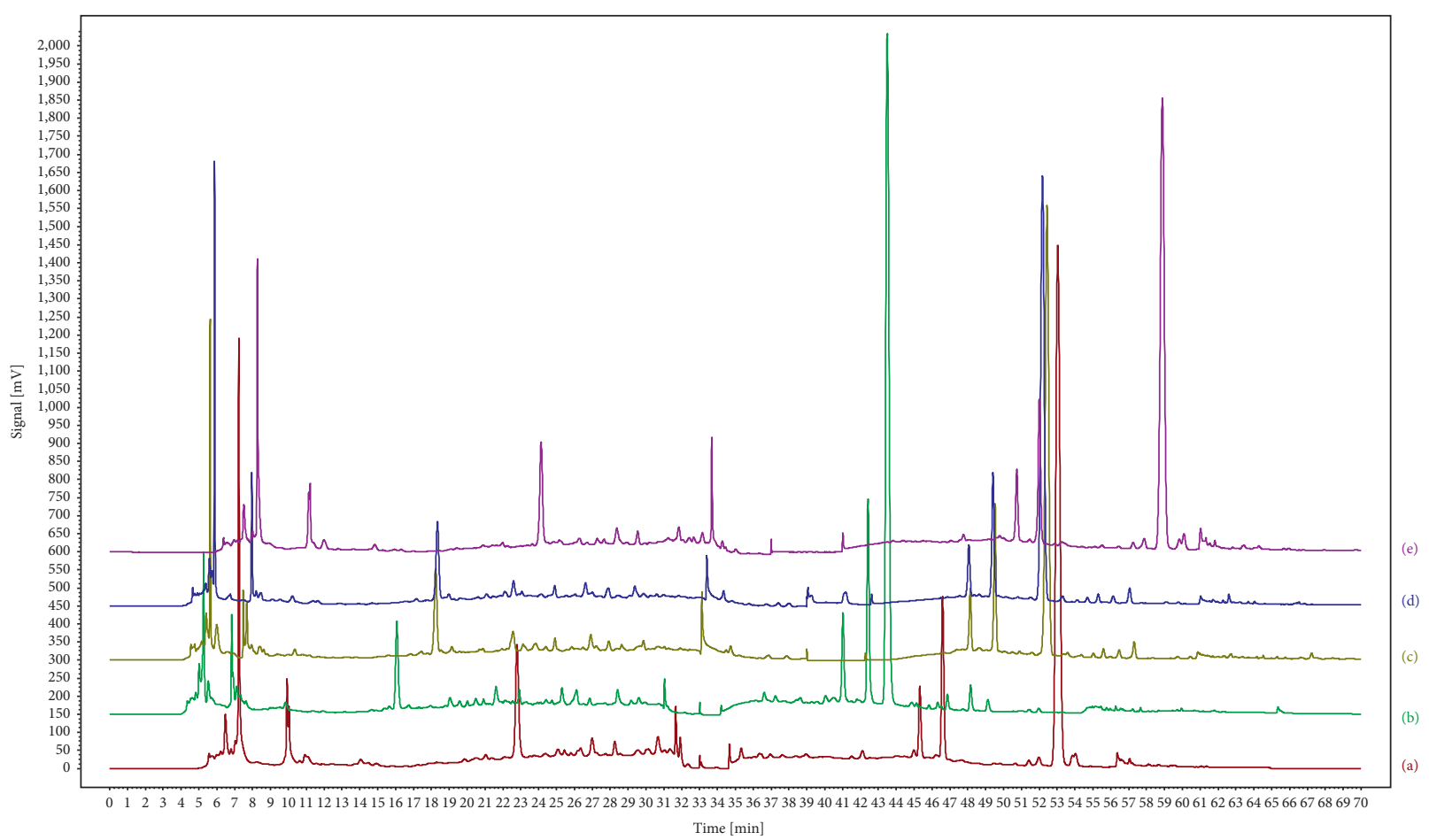

FIGURE 2: Separation results of different coupled columns by column switching technology. (a) Phenomenex Synergi Hydro-RP C18 (4 $\mu \mathrm{m}$, $250 \times 4.6 \mathrm{~mm})$ and JADE-PAK C8 $(3.5 \mu \mathrm{m}, 50 \times 4.6 \mathrm{~mm})$, (b) ACE EXCEL C18 $(5 \mu \mathrm{m}, 250 \times 4.6 \mathrm{~mm})$ and JADE-PAK C8 $(3.5 \mu \mathrm{m}$, $50 \times 4.6 \mathrm{~mm})$, (c) JADE-PAK C8 $(5 \mu \mathrm{m}, 150 \times 4.6 \mathrm{~mm})$ and JADE-PAK ODS-AQ $(5 \mu \mathrm{m}, 150 \times 4.6 \mathrm{~mm})$, (d) JADE-PAK C8 $(5 \mu \mathrm{m}$, $150 \times 4.6 \mathrm{~mm})$ and Sharpsil-U C4 $(5 \mu \mathrm{m}, 150 \times 4.6 \mathrm{~mm})$, and (e) ACE EXCEL C18 $(5 \mu \mathrm{m}, 250 \times 4.6 \mathrm{~mm})$ and JADE-PAK C8 $(5 \mu \mathrm{m}$, $150 \times 4.6 \mathrm{~mm})$.

achieved. Different column temperatures $\left(25^{\circ} \mathrm{C}, 30^{\circ} \mathrm{C}\right.$, and $35^{\circ} \mathrm{C}$ ) were compared and the most appropriate column temperature was $25^{\circ} \mathrm{C}$ (Figure S2). According to rate theory, the flow rate can affect the plate height, and thus separation effect. Compared with different flow rates $(0.5 \mathrm{~mL} / \mathrm{min}$, $0.6 \mathrm{~mL} / \mathrm{min}$, and $0.7 \mathrm{~mL} / \mathrm{min}$ ), the best condition was $0.6 \mathrm{~mL} / \mathrm{min}$ with more peaks and better resolution (Figure S3). After testing different wavelengths $(210 \mathrm{~nm}$, $230 \mathrm{~nm}, 254 \mathrm{~nm}, 260 \mathrm{~nm}$, and $270 \mathrm{~nm}$ ), $230 \mathrm{~nm}$ was selected as the most appropriate detection wavelength because most compounds had satisfactory absorption (Figure S4).

3.3. Validation of the HPLC Fingerprint Method. The characteristic peak of chlorogenic acid (peak 14 shown in Figure 3) was selected as a reference peak, and the relative standard deviations (RSDs, \%) of relative retention time (RRT) and relative peak area (RPA) of the common peaks were used for validation of the HPLC fingerprint method, including the stability, repeatability, and intraday and interday precision. The specific results are displayed in Table S1. The stability test was performed by analysis of the same sample solution with different time intervals $(0,2,4,8$, 12 , and $24 \mathrm{~h}$ ), and the RSDs values of RRT and RPA were less than $0.75 \%$ and $4.8 \%$, respectively. The repeatability test was performed by analysis of six independent TMOL samples of the same batch, and the RSDs values of RRT and RPA were less than $0.72 \%$ and $4.8 \%$, respectively. The intraday precision test was performed by analysis of the same solution six consecutive times within one day, and the RSDs values of RRT and RPA were less than $0.81 \%$ and $4.9 \%$, respectively. The interday precision test was performed by analysis of the same solution three consecutive times over three consecutive days, and the RSDs values of RRT and RPA were less than $0.71 \%$ and $5.0 \%$, respectively. The above results showed that the established method was feasible for the chromatographic fingerprint of TMOL.

3.4. Chromatographic Fingerprinting of TMOL. Under the above optimal chromatographic conditions, the fingerprints of 12 batches samples were obtained. According to their retention times (Figure 3) and ultraviolet (UV) spectra, five peaks are identified as syringin, chlorogenic acid, calycosin 7-O-glucoside, hesperidin, and icariin, respectively. The contrast of UV spectra for the above five components is shown in Figure S5.

The fingerprints of 12 batches of samples by column switching technology were input to professional software named Similarity Evaluation System for Chromatographic Fingerprint of TCM. A reference fingerprint obtained by the average method and the sample fingerprints are both shown in Figure 3. Because the column switching technology based on a single pump was used to establish the fingerprint method, the fingerprint measurement required two injections. The reference fingerprints and TMOL fingerprints of 12 batches of the first injection (before $35 \mathrm{~min}$ ) and the second injection (after $35 \mathrm{~min}$ ) are shown in Figures S6 and S7, respectively. 


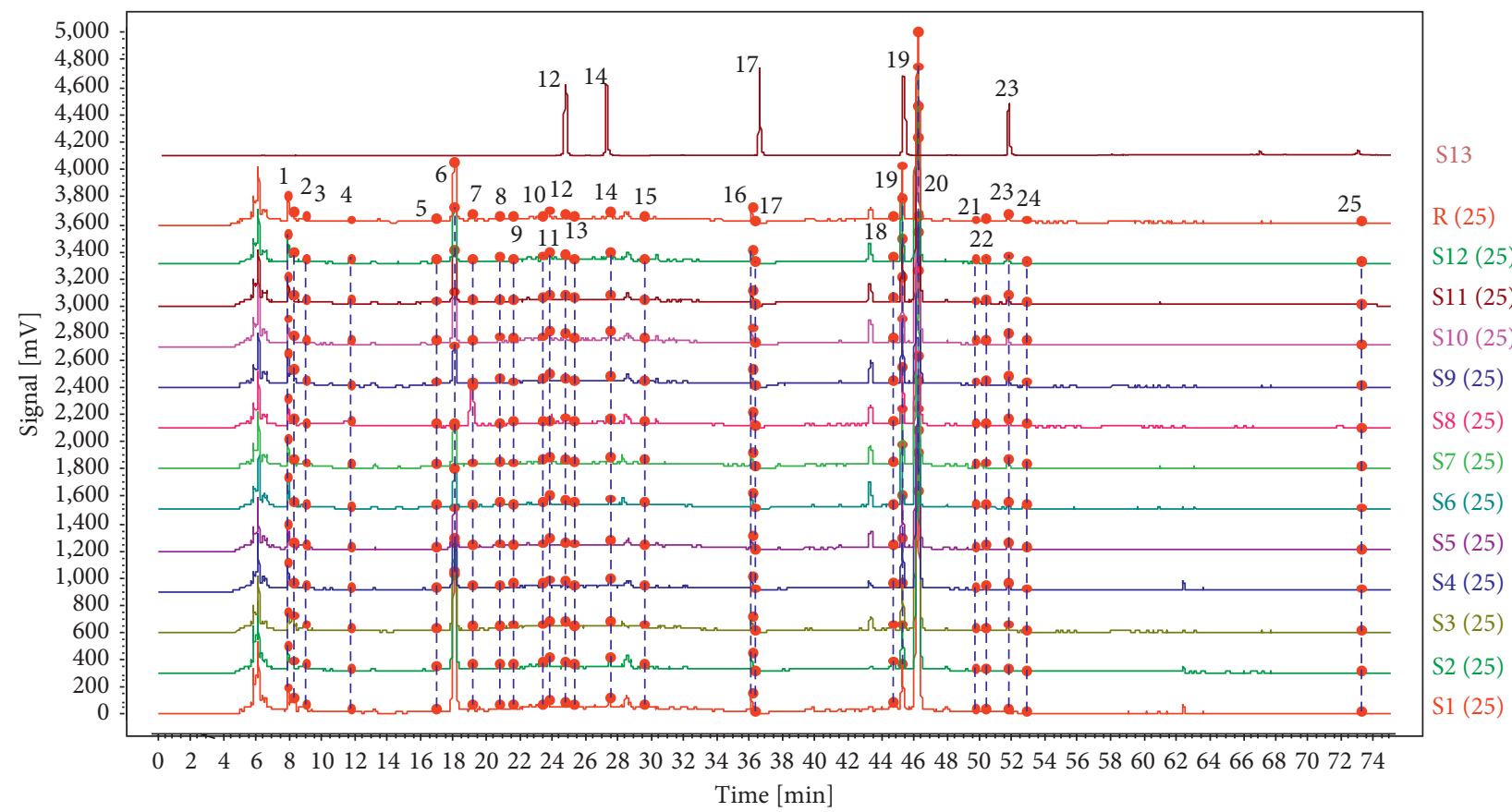

Figure 3: The fingerprints of 12 batches of TMOL, $\mathrm{R}$ reference fingerprint based on column switching technology, and (S13) the chromatogram of mixed reference standard solution. Peaks 12, 14, 17, 19, and 23 refer to syringin, chlorogenic acid, calycosin 7-O-glucoside, hesperidin, and icariin, respectively.

3.5. Similarity Analysis. Based on obtained common peaks, similarity analysis was performed between the reference fingerprint and the fingerprints of TMOL samples using MATLAB R2019a. The similarities were evaluated with the cosine method. The similarity of 10 unexpired samples were 0.999, 0.999, 0.999, 0.999, 0.998, 0.999, 0.997, 0.996, 0.996, and 0.998 , and they shared similar chromatographic patterns with similarity values higher than 0.995 . The two expired samples had lower similarity values, which were 0.978 and 0.984 , suggesting the differences existed in quality between the expired and the unexpired TMOL.

The similarity threshold is the lowest limit of fingerprint similarity for a class of samples. Interval estimation is often used to determine the threshold in statistics, which requires that the overall distribution of the data is known or approximately empirical. The bootstrap method proposed by Professor Efron in 1979 is based on the existing small amount of sample data and extends to a large amount of sample data by replacement sampling [22]. Therefore, according to the results of similarity evaluation of 12 batches of samples, the bootstrap method was used to determine the identification threshold of the unexpired TMOL samples (the number of bootstrap samples to draw is 10000, $\alpha=0.05$ ). After calculation, the similarity threshold is 0.992 . The results showed that the similarity of unexpired samples (S3S12) was higher than 0.992 , which could be considered similar and belong to the same category, while the similarity of expired samples (S1-S2) was lower than 0.992, which could be considered to be different from the quality of unexpired samples. The HPLC fingerprinting combined with similarity analysis and threshold determination could judge the quality discrepancy of TMOL samples.
3.6. Principal Component Analysis. PCA is a multivariate analysis technique widely used for feature extraction and dimensionality reduction to investigate the correlation between multiple variables [23]. The areas of 25 common peaks in chromatographic fingerprints as variables and 12 samples as objects were employed to form the data matrix, which was analyzed directly with PCA using MATLAB R2019a. Based on the eigenvalues and contribution rate of principal components, two principal components (PC1, PC2) were extracted to account for $87.1 \%$ and $10.9 \%$ of the 25 data variance in 12 batches of samples, respectively, and could well represent most information in the fingerprints of samples. The PCA score plot is shown in Figure S8. Twelve batches of TMOL samples distribute in two domains, which are labeled as group 1 (S1, S2) and group 2 (S3-S12), respectively. The boundary between the two categories is apparent. S1 and S2 are in positive part on PC1, while the rest of the samples (S3-S12) form an independent cluster with scores close to zero or negative values on PC1. On PC2, the scores of samples (S1, S2, S4, S7, and S11) are negative values, while other samples have positive scores.

Furthermore, to identify the variables causing the significant category separation, the loading plot for PC1 and PC2 is also shown in Figure S9. From the loading plot, peak 20 and peak 6 significantly contribute to the two categories. Peak 18 and peak 19 moderately lead to separation, while other peaks have little contribution to classification.

3.7. HCA with Heatmaps. In order to further clarify the influence of common peaks on classifiers, HCA with heatmaps was performed to group TMOL samples into different 


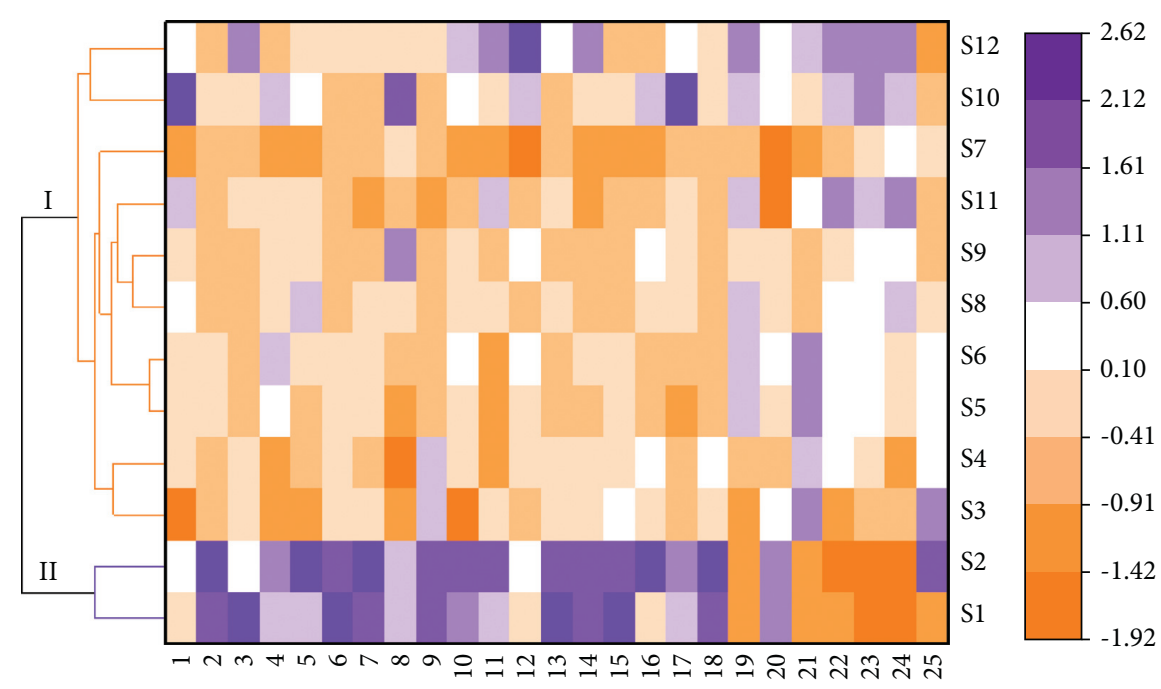

FIGURE 4: Hierarchical clustering analysis (HCA) with heatmaps based on the common peak areas for 12 batches of TMOL fingerprints.

clusters. The peak areas of 12 batches of TMOL samples were normalized, and the cluster dendrograms were generated based on Euclidean distance with group average method for measuring the dissimilarity. Visibly, all TMOL samples were gathered into two clusters (Figure 4). Specifically, the expired samples 1 and 2 are located near to each other in one cluster, and the unexpired samples 3-10 are involved in another cluster. In addition, some peaks like $6,20,18$, and 19 seem to have an obvious difference in peak area between the two clusters, which corresponds to the result obtained by the loading plot (Figure S9) of PCA. The results indicated that the fingerprints combined with HCA realized the identification of the expired and the unexpired TMOL samples.

3.8. Comparison with Fingerprinting Based on a Single Column. Three batches of TMOL samples (180101, 180104, and 180203) and the solution of the mixed standards were analyzed under the chromatographic conditions of the fingerprinting method based on a single column and column switching technology with one pump, respectively. HPLC fingerprints of 3 batches of TMOL samples and the chromatogram of mixed reference standard solution with the singlecolumn method are shown in Figure S10. The peak capacities of the three batches of samples analyzed by the single-column method were 45,46 , and 46 , while the corresponding peak capacities increased to 56,63 , and 62 by the column switching method, respectively. The column switching method could obtain a higher peak capacity than the single-column method (at least 11 chromatographic peaks) under the similar conditions. In addition, compared with the single-column method, the column switching method could increase the number of theoretical plates of the same component (Table S2) and achieve a better resolution of the five components (Table S3). Resolution (R) can be expressed as $R=(\sqrt{N} / 4)(\alpha-1 / \alpha)\left(k_{2} / k_{2}+1\right)$, and theoretical plate number $(N)$, separation factor $(\alpha)$, and retention factor of the latter peak $\left(k_{2}\right)$ are three main parameters affecting resolution. Compared with the single column, the length of the column is increased by the column switching technology, which can acquire a higher theoretical plate number. Under the condition of the same mobile phase, the change of column stationary phase affects $\alpha$ and $k_{2}$. Therefore, the improvement of resolution by column switching is attributed to the integrated effect of $N, \alpha$, and $k_{2}$.

As a comparison, there were 25 common peaks (Figure 3) in the fingerprints of different batches, including 16 peaks (Figure S6) in the first injection analysis and 9 peaks (Figure S7) in the second injection analysis, while 16 common peaks (Figure S11) were only obtained if we used a single C18 column for analysis [6] under similar measurement time. More common peaks occurred in the fingerprints, indicating more separation power with column switching technology than a single column, and could reflect chemical composition more comprehensively, which is highly meaningful for the quality evaluation of TMOL.

Traditional Chinese medicine (TCM) is different from chemically synthesized drugs in that its chemical composition is complex and the sources are extensive, making it difficult to control and evaluate its quality. HPLC fingerprinting technology is to separate the complicated chemical composition of TCM with chromatographic column under the optimal chromatographic conditions to acquire the corresponding chromatogram as fingerprint. The characteristic peaks of the fingerprint are the manifestation of the chemical composition of TCM, which can indicate its quality. Therefore, the fingerprint plays a key role in quality control and evaluation of TCM. The more peaks in fingerprint, the stronger characterization of TCM's quality. Although the column switch using a single pump due to laboratory conditions has the limited improvement on the HPLC fingerprinting separation, it would be still an alternative method to develop the fingerprint, which could have a certain positive point in quality evaluation of TCM.

\section{Conclusions}

A reliable fingerprinting method based on column switching technology by using a single pump was developed and 
applied successfully to analyze 12 batches of very complex preparation of TMOL in the present work. Through chemometric methods including similarity analysis, PCA, and HCA, the discrimination of expired and unexpired TMOL samples was successfully achieved. Column switching technology with a pump would make fingerprints have better separation power and higher peak capacity and could provide an effective method to establish fingerprints for quality control and evaluation of complex TCM preparations like TMOL.

\section{Data Availability}

The data used to support the findings of this study are available from the corresponding author upon request.

\section{Conflicts of Interest}

The authors declare that they have no conflicts of interest.

\section{Authors' Contributions}

Meng Yuan contributed to software, data curation, and manuscript review and editing. Xiaoyan Liu carried out methodology and writing of original draft. Yue Sun contributed to validation. Linlin Wang performed formal analysis. Pian Jin carried out investigation. Xiaoqi Zhuang provided resources. Hengchang Zang was responsible for supervision. Zhonghu Zhang performed visualization. Lei Nie was responsible for conceptualization, project administration, and funding acquisition. Meng Yuan and Xiaoyan Liu contributed equally to this work.

\section{Acknowledgments}

This work was supported by the Shandong Provincial Natural Fund Project (no. ZR2020MH409), Development Project of Qinghai Provincial Key Laboratory (2020-ZJ512T05), Study on the Spectral Behavior of Hyaluronic Acid Water (1310019007), and National Key R\&D Plan "Key Technology and Demonstration Research on Advanced Manufacturing of Traditional Chinese Medicine Oral Preparations" (2019YFC1711203).

\section{Supplementary Materials}

The following materials are included in the supplementary materials. Figure S1: separation results of different acids as additives. Figure S2: separation results of different column temperatures. Figure S3: separation results of different flow rates. Figure S4: separation results of different detection wavelengths. Figure S5: contrast of UV spectra for (A) syringin, (B) calycosin 7-O-glucoside, (C) calycosin 7-Oglucoside, (D) hesperidin, and (E) icariin in (a) TMOL and (b) the mixed reference standard solution. Figure S6: the fingerprints of 12 batches of TMOL and the reference fingerprinting (the first injection analysis). Figure S7: the fingerprints of 12 batches of TMOL and the reference fingerprinting (the second injection analysis). Figure S8: PCA two-dimensional score plot of 12 batches of TMOL. The number refers to the sample number. Figure S9: two-dimensional loading plot of the 25 common fingerprinting peaks. The number refers to the peak number. Figure S10: the HPLC fingerprint of (a) 3 batches of TMOL samples and (b) the chromatogram of mixed reference standard solution based on the single-column method. Peaks 1-5 refer to syringin, chlorogenic acid, calycosin 7-O-glucoside, hesperidin, and icariin, respectively. Figure S11: the fingerprints of 12 batches of TMOL and reference fingerprint based on the single-column method. Table S1: the results of precision, repeatability, and stability tests of fingerprinting method validation. Table S2: the theoretical plate number $(N)$ of the five components of two fingerprinting methods. Table S3: the resolutions $(R)$ of the five components of two fingerprinting methods. (Supplementary Materials)

\section{References}

[1] D. Xiao, "Clinical observation of Tianmeng Oral Liquid combined with eszopiclone in treatment of post-stroke insomnia," Drugs \& Clinic, vol. 31, no. 10, pp. 1612-1615, 2016.

[2] J. Zhao, M. Su, and Q. Fang, "Clinical observation of Tianmeng Oral Liquid in treatment of chronic obstructive pulmonary disease and insomnia," Chinese Traditional and Herbal Drugs, vol. 48, no. 17, pp. 3587-3589, 2017.

[3] Y. Liang, P. Xie, and F. Chau, "Chromatographic fingerprinting and related chemometric techniques for quality control of traditional Chinese medicines," Journal of Separation Science, vol. 33, no. 3, pp. 410-421, 2010.

[4] Z. Shu, X. Li, K. Rahman, L. Qin, and C. Zheng, "Chemical fingerprint and quantitative analysis for the quality evaluation of Vitex negundo seeds by reversed-phase high-performance liquid chromatography coupled with hierarchical clustering analysis," Journal of Separation Science, vol. 39, no. 2, pp. 279-286, 2016.

[5] H. Wang, M. Chen, J. Li, Y. Chang, Z. Dou, and Y. Zhang, "Quality consistency evaluation of Kudiezi Injection based on multivariate statistical analysis of the multidimensional chromatographic fingerprint," Journal of Pharmaceutical and Biomedical Analysis, vol. 177, Article ID 112868, 2020.

[6] X. Liu, H. Zhang, and M. Su, "Comprehensive quality evaluation strategy based on non-targeted, targeted and bioactive analyses for traditional Chinese medicine: Tianmeng oral liquid as a case study," Journal of Chromatography A, vol. 1620, Article ID 460988, 2020.

[7] D. Ge and Z. Deng, "Determination of astragaloside in Tianmeng capsules by HPLC-ELSD," China Pharmaceuticals, vol. 24, no. 5, pp. 31-32, 2015.

[8] X. Ma and W. Qu, "HPLC determination of the contents of strychnine in Tianmeng capsules," Herald of medicine, vol. 27, no. 4, pp. 467-468, 2008.

[9] X. Gao, H. Cao, and P. Wang, "HPLC determination of the contents of syringin in Tianmeng capsules," Chin J Pharm Anal, vol. 27, no. 12, pp. 1953-1955, 2007.

[10] F. Gao, Z. Xu, W. Wang et al., "A comprehensive strategy using chromatographic profiles combined with chemometric methods: application to quality control of Polygonum cuspidatum Sieb. et Zucc," Journal of Chromatography A, vol. 1466, pp. 67-75, 2016.

[11] X. Lu, W. Tong, S. Wang et al., "Comparison of the chemical consituents and immunomodulatory activity of ophiopogonis radix from two different producing areas," Journal of Pharmaceutical and Biomedical Analysis, vol. 134, pp. 60-70, 2017. 
[12] W. Jiang, X. Liu, M. Su, H. Zang, Y. Zeng, and L. Nie, "Chromatographic fingerprint based on serially coupled columns combined with multiple-component quantitation with a single reference standard for quality evaluation of Shen-Zhi-Ling oral liquid," Analytical Methods, vol. 11, no. 3, pp. 367-374, 2019.

[13] F. P. Prencipe, V. Brighenti, M. Rodolfi et al., "Development of a new high-performance liquid chromatography method with diode array and electrospray ionization-mass spectrometry detection for the metabolite fingerprinting of bioactive compounds in Humulus lupulus L," Journal of Chromatography A, vol. 1349, pp. 50-59, 2014.

[14] Y. Liu, Z. Liu, G. Sun, Y. Wang, J. Ling, and J. Huang, "Monitoring and evaluating the quality consistency of compound bismuth aluminate tablets by a simple quantified ratio fingerprint method combined with simultaneous determination of five compounds and correlated with antioxidant activities," PloS One, vol. 10, no. 3, Article ID e118223, 2015.

[15] S. Liu, G. Zhang, Y. Qiu et al., "Quality evaluation of Shenmaidihuang Pills based on the chromatographic fingerprints and simultaneous determination of seven bioactive constituents," Journal of Separation Science, vol. 39, no. 23, pp. 4557-4567, 2016.

[16] D. R. Stoll, X. Li, X. Wang, P. W Carr, S. E Porter, and S. C Rutan, "Fast, comprehensive two-dimensional liquid chromatography," Journal of chromatography. A, vol. 1168, no. 1-2, pp. 3-2, 2007.

[17] M. Kula, D. Głód, and M. Krauze-Baranowska, "Two-dimensional liquid chromatography (LC) of phenolic compounds from the shoots of Rubus idaeus 'Glen Ample' cultivar variety," Journal of Pharmaceutical and Biomedical Analysis, vol. 121, pp. 99-106, 2016.

[18] M. Iguiniz and S. Heinisch, "Two-dimensional liquid chromatography in pharmaceutical analysis. Instrumental aspects, trends and applications," Journal of Pharmaceutical and Biomedical Analysis, vol. 145, pp. 482-503, 2017.

[19] K. Zhao, D. Yang, and Z. Wang, "Column-switching high performance liquid chromatography and its application to food inspection," National Institute for Nutrition and Food Safety, vol. 21, no. 2, pp. 156-161, 2009.

[20] M. Hädener, P. S. Bruni, W. Weinmann, M. Frübis, and S. König, "Accelerated quantification of amphetamine enantiomers in human urine using chiral liquid chromatography and on-line column-switching coupled with tandem mass spectrometry," Analytical and Bioanalytical Chemistry, vol. 409, no. 5, pp. 1291-1300, 2017.

[21] C.-L. Hsieh, P.-Y. Lin, F. Akita et al., "Development of a threedimensional HPLC system for the simultaneous determination of lactate and 3-hydroxybutyrate enantiomers in mammalian urine," Chromatography, vol. 40, no. 1, pp. 25-32, 2019.

[22] B. Efron, "Rietz lecture-bootstrap methods-another look at the jackknife," Annals of Statistics, vol. 7, no. 1, pp. 1-26, 1977.

[23] W. Zhao, F. Sun, J. Cui, Y. Ren, M. Liu, and F. Zhao, "Quantitative analysis and chromatographic fingerprinting of WeiChangShu tablet using high-performance liquid chromatography combined with chemometric methods," Chromatographia, vol. 81, no. 9, pp. 1293-1304, 2018. 